Copyright (C) 2021 The Author/s

This work is licensed under a CC-BY 3.0 License

Peer review method: Double-Blind

Accepted: September 16, 2021

Published: November 23, 2021

Original scientific article

DOI: https://www.doi.org/10.47305/JLIA2137191d

\title{
DIGITAL TECHNOLOGY AND HEALTH ADVOCACY ON COVID-19: A CASE STUDY OF TWITTER HANDLES OF THE WORLD HEALTH ORGANIZATION AND MINISTRY OF HEALTH OF INDIA
}

\author{
Rajesh Das \\ The University of Burdwan, West Bengal, India \\ ORCID iD: https://orcid.org/0000-0002-2493-2027 \\ rd.icssr@gmail.com \\ Ipsita Banerjee* \\ ICSSR-IMPRESS (MHRD), the University of Burdwan, West Bengal, India \\ ORCID iD: https://orcid.org/0000-0002-0435-7387 \\ ipsitabanerjee95@gmail.com
}

\begin{abstract}
The article has intended to study the action of Twitter-based media advocacy promoted by the Ministry of Health $(\mathrm{MOH})$ of the Government of India, and World Health Organization (WHO) during the Covid-19 pandemic. Its goal was to assess the degree of the WHO and MOH's media campaigning for Covid-19, as well as the public's perception of this advocacy. In this regard, mixed methods have been used for data collection where a survey has been conducted with 125 respondents, who use Twitter, from Kolkata (India) with the help of random sampling. A content analysis of two well-known Twitter accounts was conducted, which helped to reflect the current trends that they follow. The findings of this research have reflected the choice of medium preferred by the respondents for receiving news and information during the Covid-19 pandemic. It has also helped to identify the Twitter handles and tweets they mostly follow and thereby the major factors influencing their choice. The outcome of this research has helped to study whether Twitter can be used for institutionalized health communication or not in the future.
\end{abstract}

Keywords: Digital Communication; Twitter; Health Advocacy; WHO; Ministry of Health; Covid-19; India

\section{INTRODUCTION}

People from throughout the world will see an unprecedented pandemic in 2020. In this situation, we saw the Indian government put in an uncompromising effort to fight the pandemic through inclusive networking within the country and around the world.

Twitter, as the third most popular social media, provides a new ecosystem of public health literacy discussion and sharing attitudes, such as the Covid-19 pandemic. 
The 140 character's format is an exceptional form in any communication research - to collect required data and to navigate a new form of interaction with audiences. It is a digital mode of communication with its openness, starlight dealings, and a high degree of authenticity in the information dissemination process. Considering the number of Twitter users, India is the third leading country in the world (Statista 2021). Health communication is a multidisciplinary approach that covers five modalities, like exchange of information, message, an act of communication, and rapport (McQuail 2005). These catalysts will help to give an associative approach to health communication with public health policy, prevention communication, and health education. According to Vilbert (2021) during and after the Covid-19 pandemic, it will be critical to assess the ability of healthcare organizations such as the World Health Organization (WHO) to provide scientific approaches to global health advocacy. Twitter, as the third most popular and engaged social media channel, enables a new ecosystem of the health promotion campaign on Covid-19. Health communication, like other forms of communication, should be built on a two-way exchange of information based on a common set of indicators and behaviors. Furthermore, public health communication should be accessible and foster a sense of shared empathy, understanding, and sympathy among members of the communication team as well as the intended audiences (Schiavo 2013). The current study looked at how major health organizations such as the World Health Organization (WHO) and the Government of India's Ministry of Health and Family Welfare (Ministry of Health; $\mathrm{MOH}$ ) use Twitter handles to disseminate health information, build relationships, and encourage people to take action to improve their health.

This study has once again contributed to a better knowledge of how a national government and an international organization maintain the digitized message design across different forms of information on the same Covid-19 aspects. As a result, it's essential to conduct a comparative study of the MOH's and WHO's approach to digital communications and their content on public health awareness.

In the event of an emergency, this form of comparative study has recommended how the national government and international organizations must work together to produce and disseminate health and hygiene communication messages. Social media like Twitter enable the public sharing of information with a high degree of transparency. The sharing of information on Twitter can create a communicative and collaborative atmosphere for patients, physicians, and researchers and even improve the quality of care. However, risks involved with using Twitter for healthcare discourse include high rates of misinformation, difficulties in verifying the credibility of sources, overwhelmingly high volumes of information available on Twitter, concerns about professionalism, and the opportunity cost of using physician time. Ultimately, the use of Twitter in healthcare can allow patients, healthcare professionals, and researchers to be more informed, but specific guidelines for appropriate use are necessary. 


\section{LITERATURE REVIEW AND RESEARCH GAP}

According to Singh (2021), the Ministry of Health focuses on three major strategies namely, communication strategy, components of the advocacy strategy, and strategies of the capacity of health. These strategies will build up a communicationbased liaison with a specific group of stakeholders. In this edited volume, a separate chapter reads as 'Managing Digital Media' that includes key actions at state level digital media advocacy and use of WhatsApp for proactive messaging. At the same time, on their official website, the WHO has notified a regular announcement on Coronavirus about its symptoms, prevention, travel advisory, and common FAQs on Coronavirus, to collect continuous feedback from all around the globe. In this scenario, it was also observed that on an opinion page, the author also explained how to advocate effectively in the age of Covid-19. The paper commented that communicating digitally on Covid-19 comes with an alternative mode of top-down environment. This is because social media advocacy can also succeed through a bottom-up movement. Perhaps it mobilizes voices of perceived communication on Covid-19. Xing and Zhang (2021) argued that an emergency attentiveness and response plan is essential to be incorporated into the health system. Their research work was based on the circumstances of India during the Covid-19 pandemic. They commented that preparedness campaigns have helped drive out misinformation about the Corona Virus from the public. Significantly, the OECD Policy Responses to Covid-19 document (2021) has commented on the role of the government to build up trust in the vaccines and communicate about the benefits of vaccination and how to deliver the vaccine doses safely and effectively throughout the country.

From 10 million in 2013, Twitter uses no have reached around 35 million in India (Statista 2021). This kind of digitized open system may generate 'warfare' between latent and manifest information in a public sphere regarding any advocacy purpose, like health and hygiene (Hamlin 2010). Park et al. (2016) focused on approaches to public health literacy in general and in pandemic scenarios. Due to its tremendous growth in users of Twitter over the past few years, the medical professionals, national and international health organizations, and patient parties have been sharing to the world what they had studied for their opinions on public health issues (Pershad et al. 2017). Perhaps this is one of the major reasons that Twitter-based public health research gained a significant space in public health and hygiene in the recent past more specifically during the Covid19 pandemic phase. Since the early XXI century, we have witnessed a critical relationship between globalization and approaches to information and communications technology (ICT) in any development, like public health and hygiene (Hamlin 2010). It may be defined as a new structural and functional public sphere in health communication (Lubenow 2012). The XXI century digitization is the major sphere of communication. Based on this digitization it seemed that dissemination of information in the public 
sphere became smooth. Alexey (2019) explained that in any public sphere digitalization is one of the major agendas to ensure a smooth communication process. This form of communication interconnects between hardware and software networks. This is a form of technological hierarchy that builds up a 'digital communication ecology'.

The digital communication networks encapsulate a new notion of advancements and values of information in society. The 'information super highway' creates a subset of information warfare, which are purely ICT-based knowledge networks (Gripsrud and Moe 2010). This pull has been decorated with different forms of digital media like Twitter, blog, discussion site, or video hosting. If we want to investigate the difficulties and techniques of information digitization for public healthcare in general and the Covid-19 pandemic in particular, we must consider financial balance as a major factor in this phenomenon. A relationship between financial balance and digitization of the public health system is too vital to spread quality information on health and hygiene in any developing country like India, considering the societal, cultural, and economic perspective.

Macnamara (2017) explained that in contrast to interpersonal communication, public communication is associated with the communication activities taking place within a public sphere. Twitter is a networking site within a definite public domain that can be said to be used by people in that public sphere. Kappel and Holmen (2019) further argued that in the case of scientific communications taxonomy is essential to be developed in the initial stages for better dissemination of information within this paradigm. It was also opined by del Carmen (2016) that to popularize the concept of scientific communication and cater the required information to the audience it is necessary to use a set of terminology. Mahfouz (2020) pointed out that in the postglobalization era with the original nation of Twitter in 2007 'hashtags' have now become a part of linguistic characteristics of the language used in social networking sites. According to Papa et al. (2006), along with the use of new taxonomy through Twitter, in the digital public sphere, the dialectic approaches are also following contradiction, motion, totality, and praxis. Hence, the media, media policies, and public these three catalysts have formed 'mediated public life', an appearance of social life in the era of digitization Craig (2004).

In 2012, Spires and Bartlett proposed their famous model on 'digital literacy' that comprises three phases of digital content namely locating and consuming, creating, and communicating. This model explains the challenges of ICT-based education in the information age (e-learning platforms). This digital literacy needs both technical and cognitive skills.

Papa et al. (2006) explained that an organization is an instrument for social change. In the present crisis the major purpose of the organization, which may be the WHO or Ministry of Health, should provide supportive information so that a group of individuals gains control of its future. Constant interconnection and reciprocal influence 
of multiple individual and social factors give the totality of message and help to complete a dialectic action through Twitter (Singhal 2006; Singhal and Rogers 2003).

The power of an international institution like the WHO enables a social actor to influence asymmetrically the decision on various perspectives about coronavirus, of other social actor/s, like ministries or national level institutions (Castells 2011). In this possible power game in any digital information sphere, digital forms of information should be constructed of meaning based on discourses through which social actors guide their actions (Castells 2001). Health communication is a process of interaction that defines how a narrator or communication will be able to convince his/her audience in a persuasive construction. During the Covid-19 pandemic, the major portion of the information network has been dominated by digital technology. Positive perspectives of an open system ensure 'a feedback loop' between human behavior, observational evidence, and reliability of the statement. In any behavioral change in communication, like public health and hygiene, a close system knowledge network may create or increase the degree of confusion instead of public awareness in the public sphere through digitization (Gripsrud 2010).

Research Gap

From the review of existing literature, it has been observed that there is a polarity of opinions given by the authors. Some of the authors have expressed their views in favor of social media and its role in health communication while some of the others have criticized the same. Moreover, the existing researches did not focus on audience analysis to identify if they prefer to accept health communication through Twitter. An advocacy communication pattern based on social media is yet to be studied by analyzing public discourse on health risk communication like the Covid-19 pandemic. This form of media advocacy would bring new approaches that will help to cope up and emerge in the public health community in the digital media sphere. In this paper, two different paradigms, contextual media advocacy, and perceived media advocacy will help to design inclusive public policy initiatives on the Covid-19 pandemic. The research has aimed to shift the focus of media advocacy from media content to the social periphery. It has also tried to explain the changes of policies of digitalized health communication from the international level to the individual level. As a result, it has contributed to the definition of the power gap, knowledge gap, and socio-psychological gap all in one study. It was also noted that the available literature did not conduct a comparison examination of national and international organizations' tweets. The current approach, on the other hand, will design the information-sharing behavior of digital communication in which Twitter serves as an information carrier. 


\section{OBJECTIVES}

The major objectives of this research are as follows:

1. To review the use of Twitter in the Covid-19 pandemic done by the World Health Organization and Ministry of Health. This systematic review will look into the extent of the WHO and MOH's media campaigning for Covid-19.

2. To determine the difference in the content provided by the $\mathrm{WHO}$ and $\mathrm{MOH}$ and evaluate their use of taxonomy through tweets regarding the Covid-19 pandemic.

3. To review the role of Twitter in the digital public sphere as a tool for health communication during the Covid-19 pandemic.

\section{METHODOLOGY}

Primary Data Collection

This research focuses on the role of Twitter handles of the WHO and $\mathrm{MOH}$ in a specified public sphere so it was necessary to collect data from the digital medium as well as from the audience themselves. For this research, the sample population consisted of Twitter users who either regularly or occasionally use this application. Primary data was gathered through a sample survey with a sample size of 125 respondents from Kolkata, which is one of the major cities in India hit by the pandemic outbreak. Questionnaires were distributed to respondents using a Google Form, which was used to collect primary data.

Sampling

This study's sample population is made up of people residing in Kolkata who are regular Twitter users and was chosen at random. Random sampling, as is well known, decreases the scope of sampling error and helps in better data analysis. As a result, for this research, we chose a random sampling method (Wimmer and Dominick 2013). In this regard, 125 respondents were contacted via random sampling to express their opinions on the usage of Twitter as a mode of health communication over other mediums.

Research Ethics

This research adhered to ethical standards such as honesty, integrity, and secrecy and the names of the participants were kept anonymous. Because no psychologically vulnerable people were involved in this study, no other ethics committee was consulted for approval. Furthermore, special precautions have been made to ensure that only adults are permitted to participate in the study, following the guidelines prohibiting 
children from participating. The participants had taken part in a survey, and the questions had been carefully designed to ensure that no individuals or communities were harmed in any way throughout the research.

Secondary Data Collection

On a global scale, the World Health Organization's Twitter handle was chosen. On the other side, the MOH's Twitter handle was chosen to reflect health promotion initiatives in India. Both of these Twitter handles had their top 100 posts analyzed till April 28, 2021. This was done to assess the use of 'taxonomy' and its application in India during the peak of Covid-19's second wave. In addition, four important factors or hashtags have been chosen in this regard: \#mask; \#sanitizer; \#socialdistancing; and \#vaccine.

Data Analysis

Quantitative data analysis was undertaken along with a graphical depiction of the data to analyze the data collected from the survey. The data were subjected to a percentage analysis to determine the respondents' perceptions of the public sphere. To assess the qualitative data collected from Twitter handles, a content analysis of the top hundred selected posts was undertaken.

\section{RESULTS AND DATA ANALYSIS}

Descriptive Statistics

The descriptive statistics were calculated to better understand the sample variation in this research.

Valid cases = 125; cases with missing value $(\mathrm{s})=0$.
\begin{tabular}{|l|r|r|r|r|r|r|}
\hline Variable & N & Mean & S.E. Mean & Std Dev & Minimum & Maximum \\
\hline Media_Preference & 125 & 2.50 & .10 & 1.07 & 1.00 & 5.00 \\
Type_of_tweets_followed & 125 & 2.66 & .11 & 1.20 & 1.00 & 5.00 \\
Preference_For_Covid_updates & 125 & 2.26 & .09 & 1.06 & 1.00 & 6.00 \\
Source_of_health_information_through_Twitter & 125 & 2.63 & .12 & 1.37 & 1.00 & 6.00 \\
\hline
\end{tabular}

Figure 1: Descriptive Statistics (Source: Online Survey)

From the mean and standard deviation, it can be observed that there is a low sample variance of this research conducted with the 125 respondents. In this regard, it can be stated that the sample involved with this research is valid. 
In the following segments, the perceptions of the respondents have been analyzed using a graphical representation.

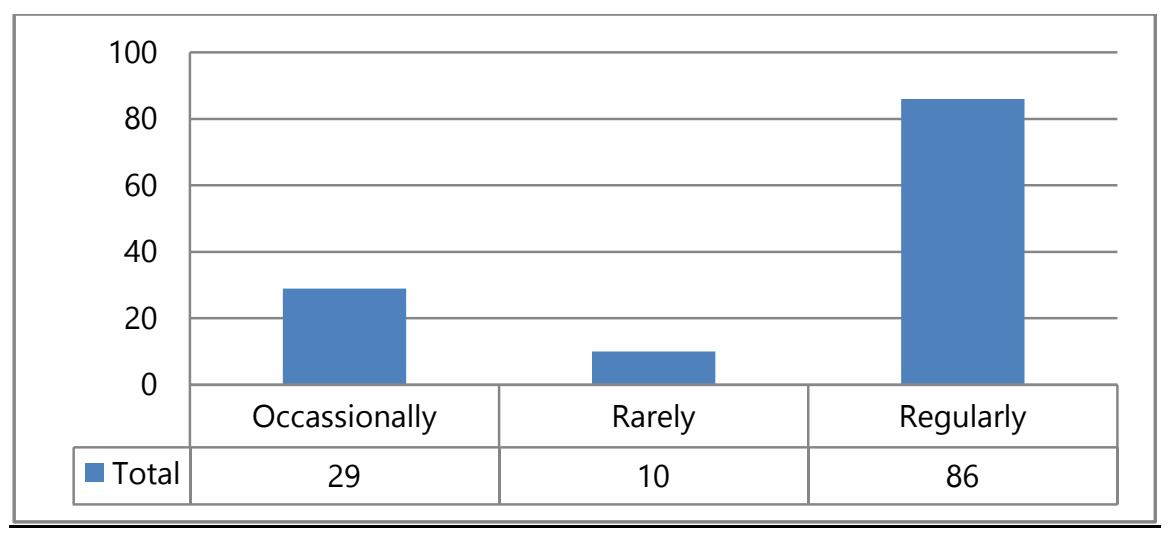

Graphic 1: Frequency of Use of Twitter (Source: Online Survey)

It can be observed that the majority of respondents, that is 86 out of 125 people, use Twitter regularly while some of them use it occasionally and only 10 out of 125 respondents use it rarely.

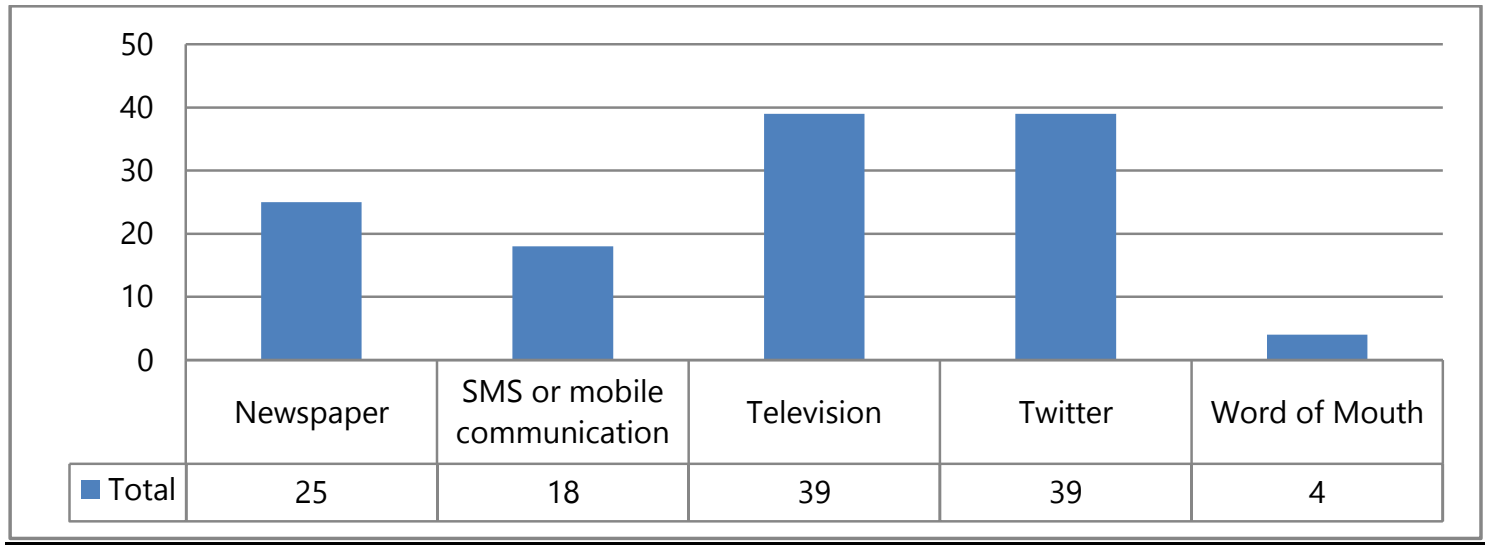

Graphic 2: Choice of Media as a Mode of Health Communication (Source: Online Survey)

In Graphic 2 it is seen that $31.2 \%$ of the respondents, that is the majority of them, have a preference for television and social media when it comes to receiving information about health communication. As a result, it can be concluded that, in addition to television, respondents prefer Twitter for health communication. 


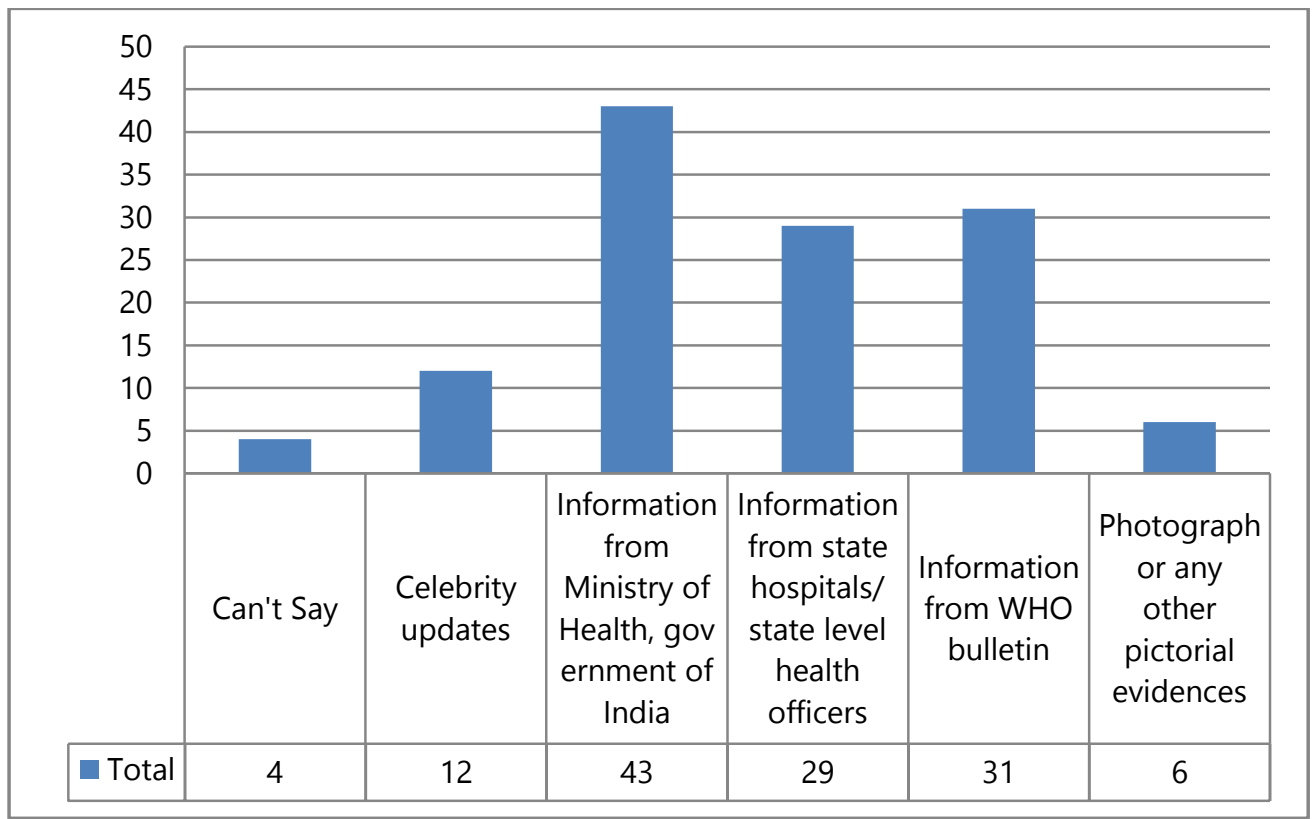

Graphic 3: Source of Information Mostly Preferred or Followed While Using Twitter (Source: Online Survey)

Finally, Graphic 3 reflects the respondents' preferences for the type of information or source of information they typically rely on while using Twitter. It is observed that the majority of respondents (35.5 percent) rely on information from the Ministry of Health of India, while the second-highest (25.6 percent) rely on information provided by the WHO on Twitter. There are also other popular information sources like information that are provided by local or state hospitals and state-level health officers through Twitter as a local information bulletin and graphical representation which is followed by some of the other respondents. 


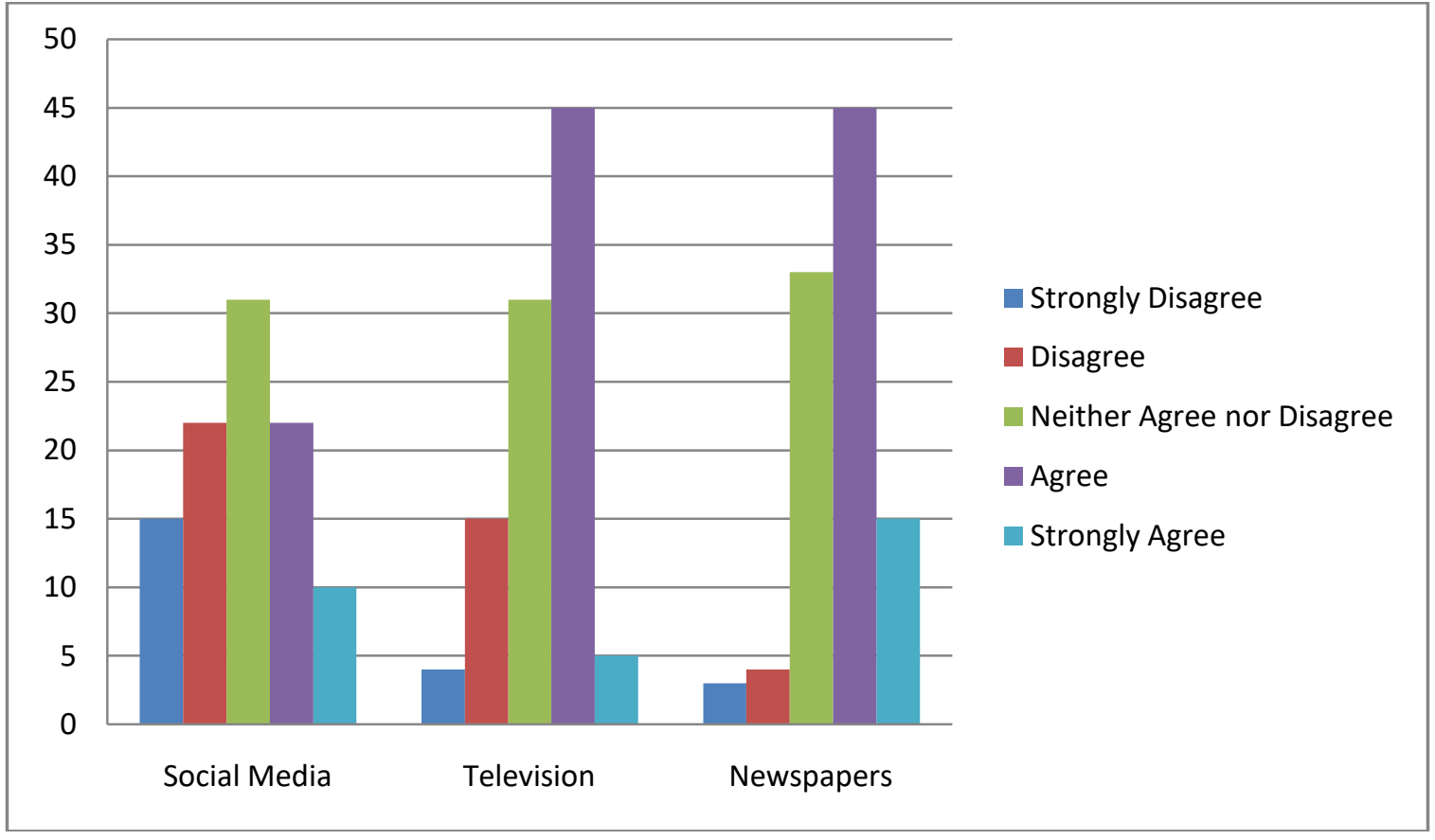

Graphic 4: Authenticity of Media for Promotion of Scientific Approaches

(Source: Online Survey)

The majority of respondents agreed with the statement that newspapers and television promote scientific approaches better than social media, especially in the case of health communication, as shown in Graphic 4. Furthermore, when asked about their attitudes regarding social media, the majority of respondents had a neutral response, indicating that they neither agree nor disagree with the statement. 


\section{TWITTER HANDLES CONTENT ANALYSIS}

\section{World Health Organization}
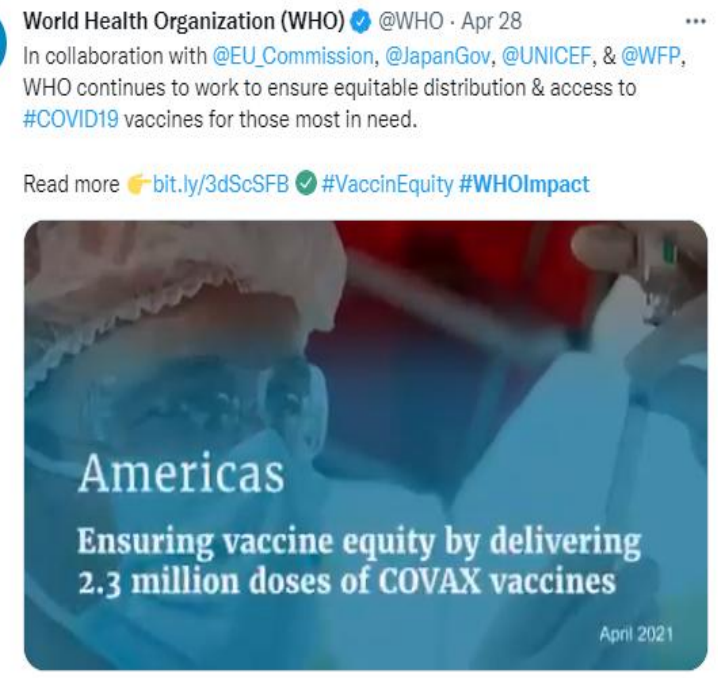
Q 7
〔】 28
○ 95
个

Image 1: Screenshot of One of WHO's Tweets Posting a Video (Source: WHO 2021)

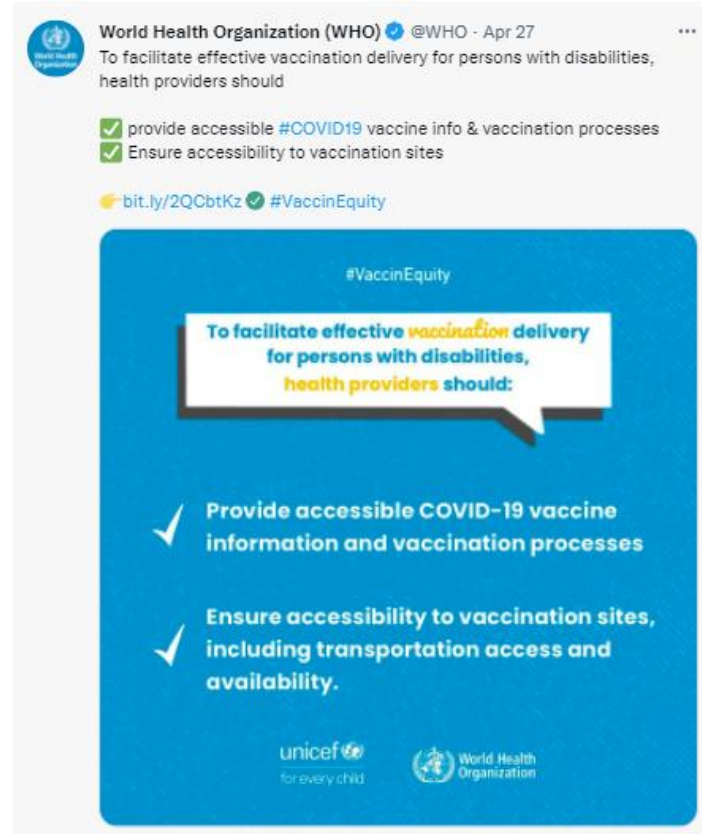

Image 2: A Tweet from WHO Representing the Vaccination Guidelines (Source: WHO 2021) 
From the Twitter handle of the WHO out of the top 100 posts, pictures of two of them have been cited above as examples. It can be observed that the WHO page has more than 60000 tweets with 9.2 billion followers. On the other hand, their approach is broad and covers a variety of countries, rather than focusing solely on India, which is technically impossible for the WHO to do. As a result, it has a proximity lock in some circumstances. The WHO tweets reflect the statistical aspects of the number of cases on the number of recoveries in a specific country. Thus, in this case, the approach is designed considering a holistic approach towards the world in general.

When it comes to word choice or taxonomy, it can be seen that the WHO primarily uses words like 'guidance' or 'guidelines' to issue any post in the public interest. In addition to this, the tweets consisted of words like 'provide' and 'ensure' which are also taxonomically related to the concept of guidelines. Whether it's the use of masks or the requirement for vaccinations, the WHO serves as a resource for the general public. In the post that was chosen for analysis, the words 'guidelines' and 'guidance' were found to be relatively freely dominating. Also, it was observed that the WHO creates more video than static graphical representations. Because the audiovisual media is believed to be more powerful than the merely visual medium, this increases the possibility of attracting an audience.

Ministry of Health

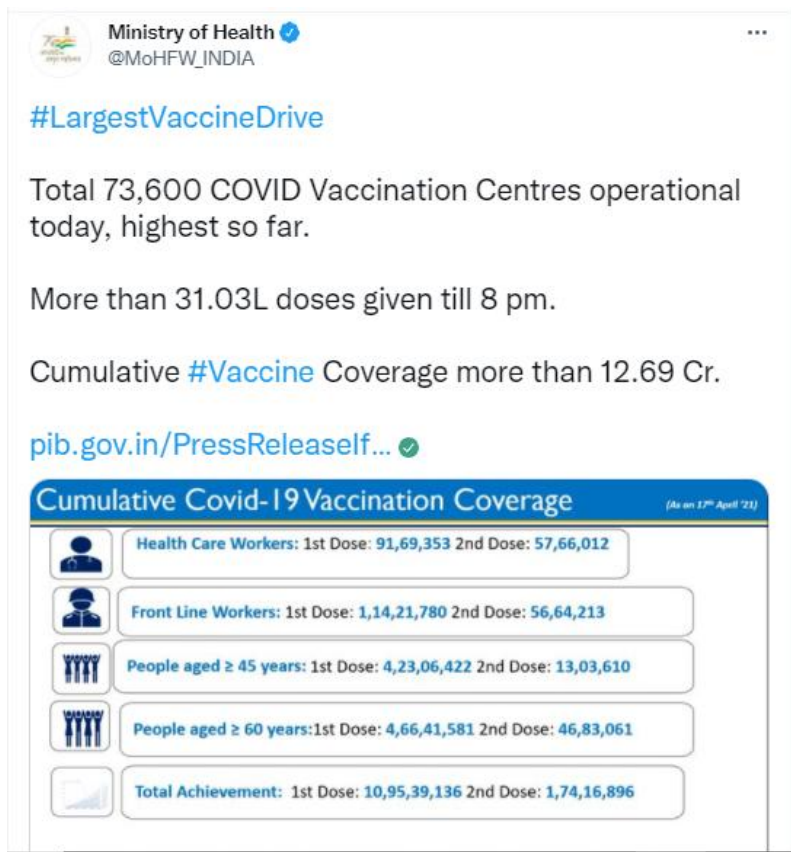

Image 3: Tweets from the Ministry of Health Representing Vaccination Statistics in India (Source: Ministry of Health 2021) 


\section{\#Unite2FightCorona}

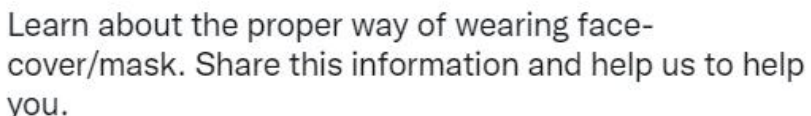
you.

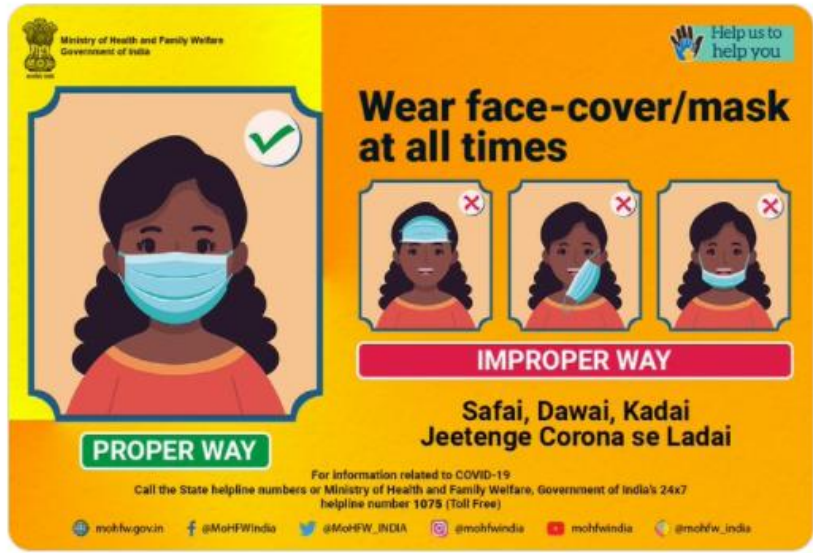

2 PMO India and 6 others

Image 4: A Picture Posted on Twitter by the Ministry of Health to Teach People How to Properly Wear Masks (Source: Ministry of Health 2021)

The Twitter handle of the Ministry of Health does not contain posts about its initiatives or awareness campaigns against Covid-19 but they also re-tweet other politicians and their perspectives. The other above figures are examples of the type of posts that are done by this Twitter handle. The first screenshot of this Ministry of Health shows the statistics of the application of vaccines in India as well as its availability. Even though this image is static and lacks audiovisual interactive design, yet it can be engaging for the Indian audience because of its proximity. In this situation, the second screenshot shows a post by the Ministry of Health to educate people on how to correctly wear masks. If the 'taxonomy' is examined, the term 'learn' has been employed to educate people about wearing masks in this situation. In this regard, it should be noted that the Ministry of Health has been able to construct tweets or posts that are easily engaging for the citizens of India.

\section{COMPARISON OF THE WHO AND MINISTRY OF HEALTH'S TWITTER HANDLES}

There are numerous discrepancies between the tweets sent by the $\mathrm{MOH}$ and those sent by the $\mathrm{WHO}$, ranging from the usage of color to the languages used. For health advocacy, the WHO has selected a formal mode of taxonomy where words like 'guidance' or 'guidelines' are mainly used. 
On the other hand, for health advocacy, the term 'learn' has been used by the $\mathrm{MOH}$ to make people educated about wearing masks. So, unlike the term 'guidelines', 'learn' is more interactive and it evokes the audience to a great extent. Moreover, the $\mathrm{MOH}$ has not only used English as a language to design their tweets but it has considerably used 'Hindi', one of the most popular Indian languages, in their tweets as well which can be easily understood by the people of India. Similarly, the WHO has utilized largely blue and white or neutral tones of color in their content, which is another formal method. On the contrary, the $\mathrm{MOH}$ has employed colorful representations that are easily comparable to India's colorful and diverse population.

\section{DISCUSSION}

From the primary data analysis, it can be observed that the respondents consisted of a mixed group where people from different age groups, gender, and educational level have taken part in the survey (see: Annex I). Some of the respondents have even suffered from Covid-19 themselves or have seen their family members suffering from this disease, unfortunately (see: Annex II). For health communication through Twitter has received the highest number of responses by measuring the preferences of the audience. Yet, it can be further observed that social media is not the only medium that has been highly preferred (Graphic 1). Rather, in this research, both social media and television obtained an equal number of responses. The majority of respondents, on the other hand, agreed that television and newspapers have a greater influence than social media in promoting scientific approaches (Graphic 4). This indicates that, in the opinion of the respondents, television and newspapers are more trustworthy than Twitter.

Based on the findings, it can be deduced that the Ministry of Health is preferred over the WHO when it comes to sharing information. In this case, proximity can be one of the major factors influencing the choice of the Twitter handle by the respondents (Schaal et al. 2012). When it comes to a pandemic situation then only propagating information and health and safety guidelines may not be sufficient to engage the public sphere (Davis 2021). From the content analysis, it was seen that audiences prefer to receive more information about their country from the $\mathrm{MOH}$ Twitter handle in comparison to the WHO. Furthermore, with content analysis of both the Twitter handles it is clear that the WHO has more followers on Twitter worldwide. They have even developed audiovisual content through videos instead of textual tweets to attract more audience yet taxonomy or the use of language is an important factor to reach out to the selected public sphere.

When the language used by the WHO in its tweets was examined, it was discovered that they were using it as an instructional form, similar to 'guidelines,' to inform people about specific norms or behaviors to follow during the Covid-19 
pandemic. On the other hand, the $\mathrm{MOH}$ lacks the proper video representation in their tweets. Nonetheless, the Ministry of Health has a more interactive vocabulary, which may make it simpler for people in subaltern and rural areas such as India to grasp and follow the content. For example, if we follow the National Healthcare Communication Programme's (2021) Twitter feed, two subjects emerge: 'Join Twitter now to discover new and interesting conversations about the things that matter most to you, like Covid19 ' and 'Covid 19 - government and public officials', have been implying an interactive participatory communication that is based on G2C (Government to Citizens) model. It was also observed that the WHO has very few posts that are focused on any specific country rather the tweets are mainly generalized and consist of information from different parts of the world.

On the other hand, it was observed that the $\mathrm{MOH}$ has provided geographical zone-wise specific information through its tweets on vaccination campaigns, vaccination coverage, and even the number of Covid-19 cases in India. This further makes it clear that proximity in content design as well as in disseminating information is crucial when it comes to promoting health communication with the help of Twitter (Bunker et al. 2020). In this case, agenda-setting theory can be said to have been applicable. McCombs et al. (2014) had emphasized that in the contemporary era network agenda setting plays a pivotal role. In contrast to the $\mathrm{WHO}$, the agenda-setting network of the $\mathrm{MOH}$ has potentially reached out to the Indian audience, that is, the public sphere analyzed in this research. Even though Salikov (2019) and Macnamara (2017) had explained the public sphere to be free from geographical boundaries, in a digital space yet this research has proved that proximity of content is more preferred by the audience in case of a pandemic. As a result, it can be stated that the MOH's Twitter handle is chosen due to its content proximity and taxonomy. It is known that taxonomy plays a vital role in making it easy for people to classify the information received from the public domain (Kappel and Holmen 2019). It can be further noted from this study that the WHO and $\mathrm{MOH}$ are equally doing health advocacy but they are not equally accepted by the audience due to factors of the proximity of content and content design through tweets.

\section{CONCLUSION}

It can be concluded that, in addition to social media, television occupies a significant position in the public realm when it comes to the preferred medium for health communication. It was also found that the inclination of the respondents is more towards local reporting than that of the global scenario when it comes to getting information about the Covid-19 pandemic. This is one of the reasons why the Ministry

of Health's Twitter handle is chosen over the WHO's. In this regard, taxonomy and the choice of language played a very crucial role along with the proximity of content that further helped to effective communication through Twitter. 
However, it is important to keep in mind that the WHO has a global perspective, which means it may not be possible for the organization to tailor its tweets to a specific country's audience. Because of the result of this research, it is recommended that, in addition to presenting a holistic structure of Twitter content, the WHO should focus on using more interactive language to appeal to its global audiences. Furthermore, in some circumstances, different media are preferable, particularly due to authenticity concerns. As a result, if authenticity and taxonomy are adequately preserved, Twitter could emerge as a powerful medium for pandemic communication. 


\section{COMPLIANCE WITH ETHICAL STANDARDS}

Acknowledgments:

Not applicable.

\section{Funding:}

Not applicable.

Statement of human rights:

All procedures performed in studies involving human participants were following the ethical standards of the institutional and/or national research committee and with the Declaration of Helsinki and its later amendments or comparable ethical standards.

Statement on the welfare of animals:

This article does not contain any studies with animals performed by any of the authors.

Informed consent:

Informed consent was obtained from all individual participants included in the study. 


\section{REFERENCES}

1. Alexey, S. (2019). The digital transformation of the public sphere, its features in the context of various political regimes, and its possible influence on political processes. Sociologičeskoe obozrenie, 18(4).

2. Bunker, D., Mirbabaie, M., \& Stieglitz, S. (2020). Convergence behavior of bystanders: an analysis of 2016 Munich shooting Twitter crisis communication. arXiv preprint arXiv:2004.08573.

3. Castells, M. (2001). The Internet galaxy: Reflections on the Internet, business, and society. Oxford University Press on Demand.

4. Castells, M. (2011). Communication power. OUP Oxford.

5. Craig, G. (2004). The media, politics, and public life. Allen \& Unwin.

6. Davis, M. (2021). The online anti-public sphere. European Journal of Cultural Studies, 24(1), 143-159.

7. del Carmen Sanchez-Mora, M. (2016). Towards a taxonomy for public communication of science activities. The Journal of Science Communication, 15(02).

8. Gripsrud, J., \& Moe, H. (2010). The digital public sphere: Challenges for media policy. Nordicom, University of Gothenburg.

9. Hamlin, J. (2010). Epistemology, Pedagogy, and Latent Functions: The Peculiar Nature of Web-Based Public Access Courses. International Journal of Teaching and Learning in Higher Education, 22(3), 357-364.

10. Kappel, K., \& Holmen, S. J. (2019). Why science communication, and does it work? A taxonomy of science communication aims and a survey of the empirical evidence. Frontiers in Communication, 4, 55.

11. Lubenow, J. A. (2012). The public sphere 50 years later: Public sphere and mass media in Jürgen Habermas. Problemata: Revista Internacional de Filosofía, 3(2), 228-274.

12. Macnamara, J. (2017). Evaluating public communication: Exploring new models, standards, and best practice. Routledge.

13. Mahfouz, I. (2020). The Linguistic Characteristics and Functions of Hashtags:\# Is it a New Language?. Arab World English Journal (AWEJ) Special Issue on CALL, (6).

14. McCombs, M. E., Shaw, D. L., \& Weaver, D. H. (2014). New directions in agendasetting theory and research. Mass communication and society, 17(6), 781-802.

15. McQuail, D. (1987). Mass communication theory: An introduction. Sage Publications, Inc.

16. Ministry of Health. (2021). Twitter. Retrieved 28 April 2021, from https://twitter.com/search?q=Ministry\%20of\%20Health\%20Government\%20of\%2 Olndia\&src=typed_query. 
17. National Health Communication Programme. (2021). Twitter. Retrieved 30 April 2021, from https://twitter.com/nhcprogramme?lang=en.

18. Papa, M. J., Singhal, A., \& Papa, W. H. (2006). Organizing for social change: A dialectic journey of theory and praxis. Sage.

19. Park, H., Reber, B. H., \& Chon, M. G. (2016). Tweeting as health communication: health organizations' use of Twitter for health promotion and public engagement. Journal of health communication, 21(2), 188-198.

20. Pershad, Y., Hangge, P. T., Albadawi, H., \& Oklu, R. (2018). Social medicine: Twitter in healthcare. Journal of clinical medicine, 7(6), 121.

21. Schaal, M., O'Donovan, J., \& Smyth, B. (2012, December). An analysis of topical proximity in the Twitter social graph. In International Conference on Social Informatics (pp. 232-245). Springer, Berlin, Heidelberg.

22. Schiavo, R. (2013). Health communication: From theory to practice (Vol. 217). John Wiley \& Sons.

23. Singh, P., 2021. [online] Mohfw.gov.in. Available at: https://www.mohfw.gov.in/pdf/Covid19CommunicationStrategy2020.pdf [Accessed 1 October 2021].

24. Spires, H., \& Bartlett, M. (2012). Digital literacies and learning: Designing a path forward. Friday Institute White Paper Series, 5.

25. Statista, 2021. Twitter: most users by country | Statista. [online] Statista. Available at: https://www.statista.com/statistics/242606/number-of-active-twitter-users-inselected-countries/ [Accessed 1 October 2021].

26. Su, L. Y. F., Scheufele, D. A., Bell, L., Brossard, D., \& Xenos, M. A. (2017). Information-sharing and community-building: Exploring the use of Twitter in science public relations. Science Communication, 39(5), 569-597.

27. Vilbert, J., 2021. Global health governance post-Covid-19: time for a hierarchical order?. Journal of Liberty and International Affairs, 7(2), pp.11-30.

28. WHO. (2021). Twitter. Retrieved 28 April 2021, from https://twitter.com/WHO?ref src=twsrc\%5Egoogle\%7Ctwcamp\%5Eserp\%7Ctwgr \%5Eauthor.

29. Wimmer, R. D., \& Dominick, J. R. (2013). Mass media research. Cengage learning.

30. Xing, C. and Zhang, R., 2021, January. COVID-19 in China: responses, challenges, and implications for the health system. In Healthcare (Vol. 9, No. 1, p. 82). Multidisciplinary Digital Publishing Institute. 


\section{Annex I: Demographic Profile of the Respondents}
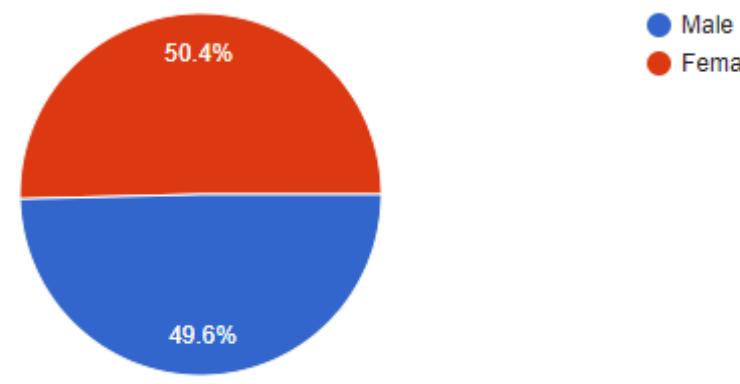

Female

Figure 2: Gender (Source: Online survey)

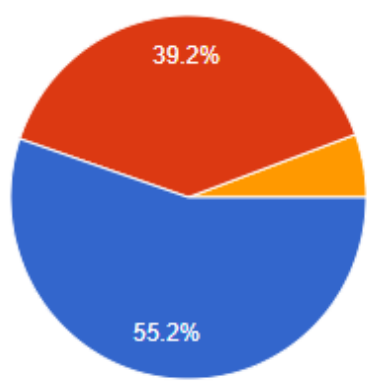

18-30 years

$31-59$ years

60 years and above

Figure 3: Age (Source: Online survey)

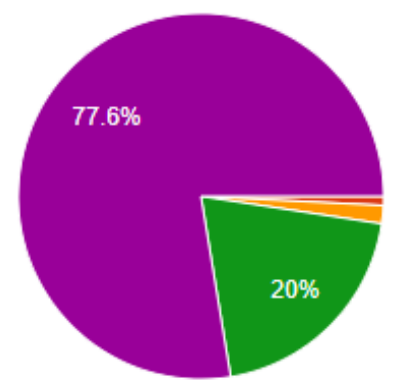

literate without formal schooling

Primary to class 8

Class 10-12

- Graduate

Post Graduate

Figure 4: Educational Qualification (Source: Online survey) 


\section{Annex II: Health Issues}

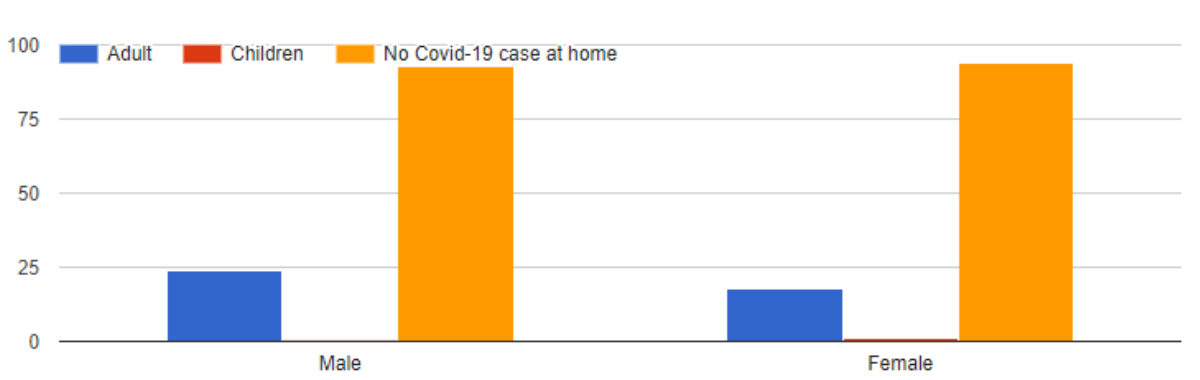

Figure 5: Number of Covid-19 Patients at Home (Source: Online survey)

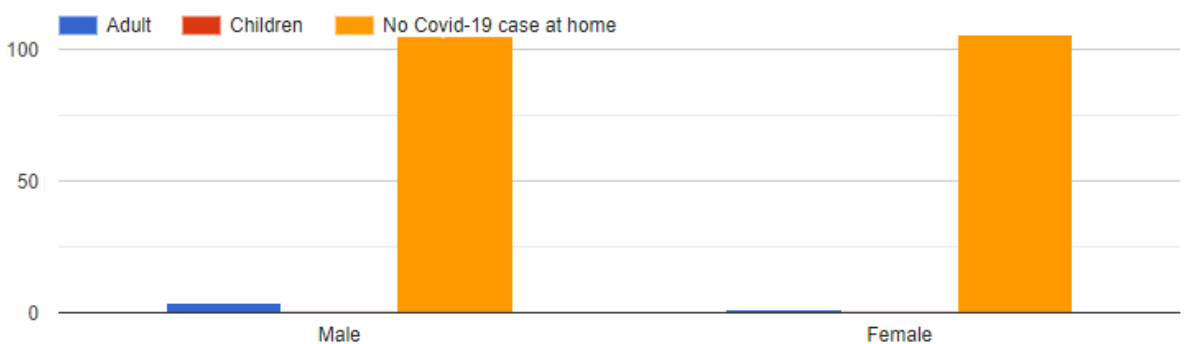

Figure 6: Number of Deaths in Family Due to Covid-19 (Source: Online survey) 